*Generalized Linear Mixed Models.

GENLINMIXED

/DATA STRUCTURE SUBJECTS=Horse*Session REPEATED_MEASURES=Trial

COVARIAN̄CE TYPE=DIAGONAL

/FIELDS TARGET=Outcome TRIALS=NONE OFFSET=NONE

/TARGET OPTIONS DISTRIBUTION=BINOMIAL LINK=LOGIT

/FIXED EFFECTS=Session Horse picture Other picture USE_INTERCEPT=TRUE

/RANDOM USE INTERCEPT=TRUE SUBJECTS=Horse

COVARIANCE TY $\bar{P} E=V A R I A N C E$ COMPONENTS SOLUTION=FALSE

/BUILD_OPTIONS TARGET_CATEGORY_ORDER=ASCENDING

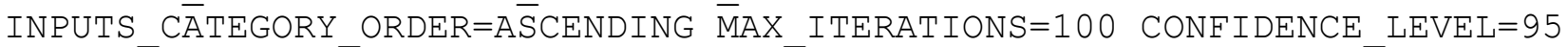

DF_METḦOD=RESIDŪAL COVB=MODEL PCONVERGE=0.000001 (ABSOLUTE) SCORING=0

$S I \bar{N} G U L A R=0.000000000001$

/EMMEANS TABLES=Session COMPARE=Session CONTRAST=PAIRWISE

/EMMEANS TABLES=Horse picture COMPARE=Horse picture CONTRAST=PAIRWISE

/EMMEANS TABLES=Other picture COMPARE=Other picture CONTRAST=PAIRWISE

/EMMEANS_OPTIONS SCALE=TRANSFORMED PADJUST=SEQSIDAK.

Generalized Linear Mixed Models

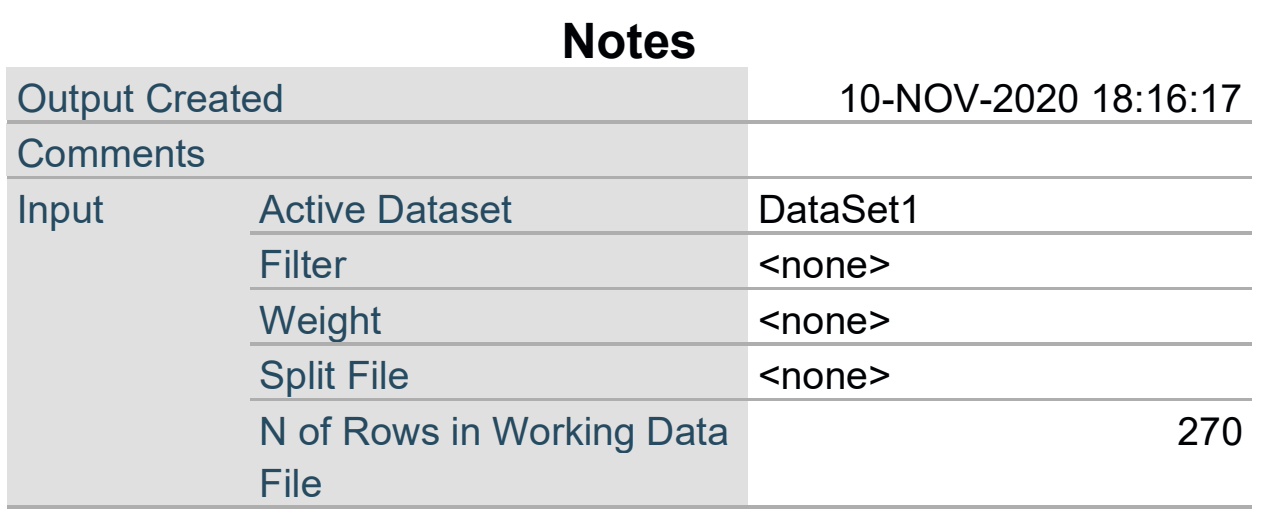

\title{
Case Processing
}

Summary

\begin{tabular}{lr|r} 
& N & Percent \\
\hline Included & 270 & $100,0 \%$ \\
\hline Excluded & 0 & $0,0 \%$ \\
\hline Total & 270 & $100,0 \%$ \\
\hline
\end{tabular}

Model Summary

\begin{tabular}{llll} 
Target & \multicolumn{2}{l}{ Outcome +/- } \\
\hline Probability Distribution & Binomial & \\
\hline Link Function & & Logit & \\
\hline Information Criterion & Akaike Corrected & & 3552,604 \\
\cline { 2 - 3 } & Bayesian & & 3590,035 \\
\hline
\end{tabular}

Information criteria are based on the -2 log likelihood $(3529,476)$ and are used to compare models. Models with smaller information criterion values fit better. 


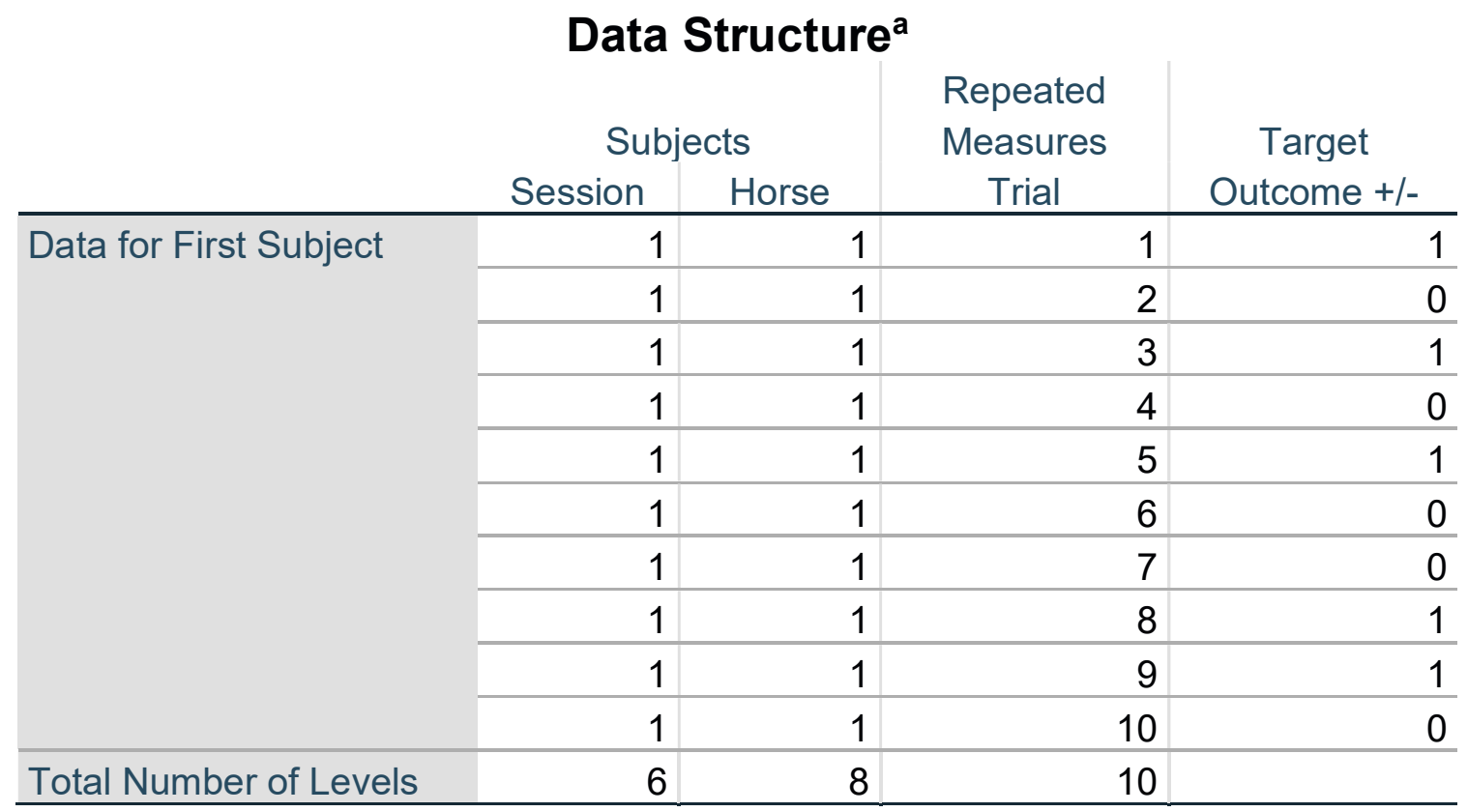

a. Target: Outcome +/-

\section{Classification \\ Overall Percent Correct $=72,6 \%^{a}$}

Predicted

\begin{tabular}{l|r|r|r}
\multicolumn{2}{l}{ Observed } & \multicolumn{1}{c|}{0} & \multicolumn{1}{c}{1} \\
\hline 0 & Count & 29 & 46 \\
\cline { 2 - 4 } & $\%$ within Observed & $38,7 \%$ & $61,3 \%$ \\
\hline 1 & Count & 28 & 167 \\
\cline { 2 - 4 } & $\%$ within Observed & $14,4 \%$ & $85,6 \%$ \\
\hline
\end{tabular}

a. Target: Outcome +/-

\section{Fixed Effects ${ }^{a}$}

\begin{tabular}{lr|r|r|r} 
Source & \multicolumn{1}{l|}{ F } & df1 & df2 & \multicolumn{1}{c}{ Sig. } \\
\hline Corrected Model &, 969 & 23 & 246 &, 507 \\
\hline Session &, 733 & 5 & 246 &, 600 \\
\hline Horsepicture & 1,234 & 9 & 246 &, 274 \\
\hline Otherpicture1 & 1,030 & 9 & 246 &, 417 \\
\hline
\end{tabular}

Probability distribution: Binomial

Link function: Logit ${ }^{a}$

a. Target: Outcome +/- 


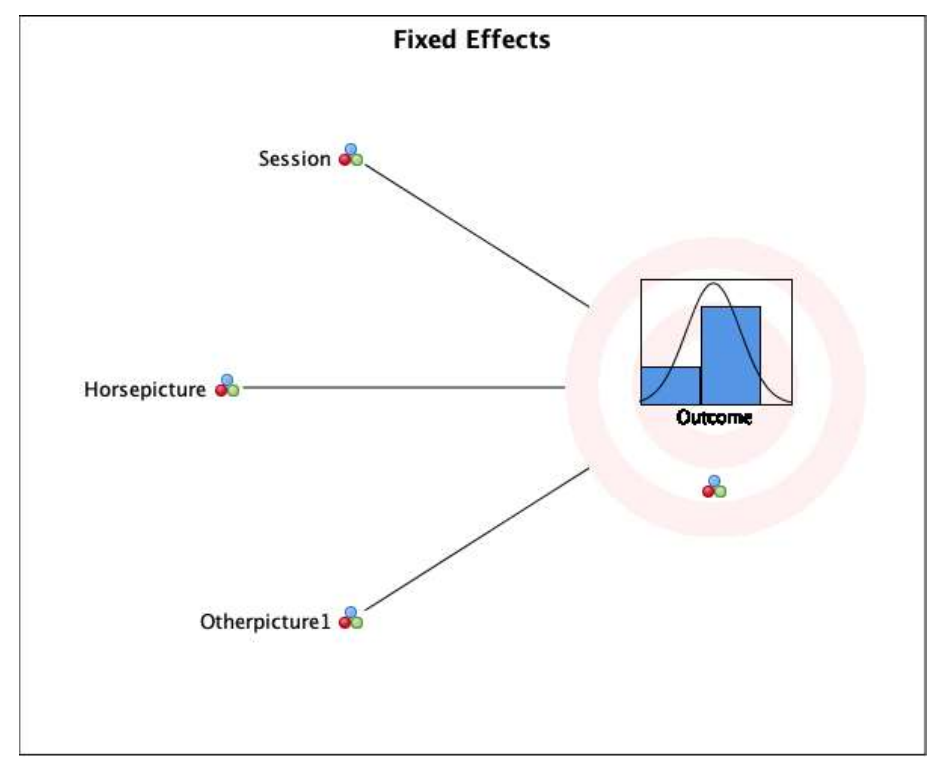

\section{Overall Test Results}

\begin{tabular}{l|rrr|r}
$F$ & df1 & \multicolumn{1}{c}{ df2 } & Sig. \\
\hline 1,030 & & 9 & 246 &, 417 \\
\hline
\end{tabular}

